\title{
Investigated Methods of Improving the Efficiency of Diagnosing Microprocessor Devices of Data Transmission Systems Based on Multi-Channel Signature Analysis
}

\author{
Rustam Xusanovich Djuraev, Jo'shqin Boltabaevich Baltaev \\ Department of Data Communication Networks and System, Telecommunication Faculty, Tashkent University of Information Technologies \\ Named After Muhammad al-Khwarizmi, Tashkent, Uzbekistan
}

Email address:

jowkin@mail.ru (J. B. Baltaev)

\section{To cite this article:}

Rustam Xusanovich Djuraev, Jo'shqin Boltabaevich Baltaev. Investigated Methods of Improving the Efficiency of Diagnosing Microprocessor Devices of Data Transmission Systems Based on Multi-Channel Signature Analysis. Communications.

Vol. 7, No. 1, 2019, pp. 13-24. doi: 10.11648/j.com.20190701.13

Received: February 5, 2019; Accepted: March 12, 2019; Published: April 13, 2019

\begin{abstract}
The article deals with tasks related to monitoring and diagnostics of microprocessor devices of data transmission systems. We have completed the life cycle of the technical means of data transmission systems and the ways of their enhanced reliability. The analysis of tasks and classification of systems of technical diagnostics of microprocessor devices of data transmission systems is carried out. The analysis of modern microprocessor devices of data transmission systems as an object of control and diagnostics necessary for the choice of characteristics of signature analysis methods is made, that the main documents of signature analysis are dictionaries of the reference signatures. Methods of calculation and determination of signatures for signature analysis in the diagnosis of microprocessor devices are considered. It is shown that the main document of signature analysis is the dictionary of reference signatures. A comparative analysis of the methods of calculation and determination of reference signatures used in the diagnosis of microprocessor devices has been performed. Algarymms and simulation programs for one and multi-channel signature methods for automating the determination of reference signatures for multi-output microprocessor devices have been developed.
\end{abstract}

Keywords: Microprocessor Devices (MPD), Life Cycle, Signature Analysis (SA), Signatura, Single-channel Signature Analysis (SSA), Multichannel Signature Analysis (MSA)

\section{Introduction}

When constructing modern data transmission systems (DTS), an urgent task is to study methods for improving the quality of operation of technical facilities and the system as a whole [1-3]. The modern DTS uses an element base based on the use of large-scale integrated circuits (SIC), ultra-large integrated circuits (LIC) and microprocessor kits (MPK) that significantly improve the efficiency of systems - increase productivity and reliability, expand the functionality of systems, dimensions and power consumption. At the same time, the transition to the widespread use of SIC, LIC and MPK in modern DTS, together with indisputable advantages, has created a number of serious problems, connected primarily with the processes of their control and diagnostics [4-7]. The main criterion for reliability of DTS is the availability factor, the components of which are the average time between failures, characterizing system reliability and the average recovery time, which characterizes its maintainability [1-3]. Since any DTS has ultimate reliability, then if there are failures in it, it becomes necessary to quickly detect, find and fix faults, and restore specified reliability indicators. In the DTS environment, the most important of the two components is the recovery time, which is determined by the detection time (monitoring) and the troubleshooting time (diagnostics), therefore one of the main activities is to improve the technical and operational characteristics of the DTS hardware by improving maintainability. This is due to the fact that one-sided orientation towards achieving high reliability of DTS to the detriment of maintainability often does not provide an increase in the availability factor in operating conditions, since the complexity of troubleshooting leads to an increase in the recovery time. 
In this regard, one of the decisive prerequisites for achieving high reliability characteristics is related to the further increase in the intensity of work on the development and use of new approaches and methods for monitoring and diagnosing DTS and their technical means at all stages of the life cycle.

\section{Life Cycle of DTS and Ways to Increase Reliability Characteristics}

The DTS life cycle is a set of research, development,

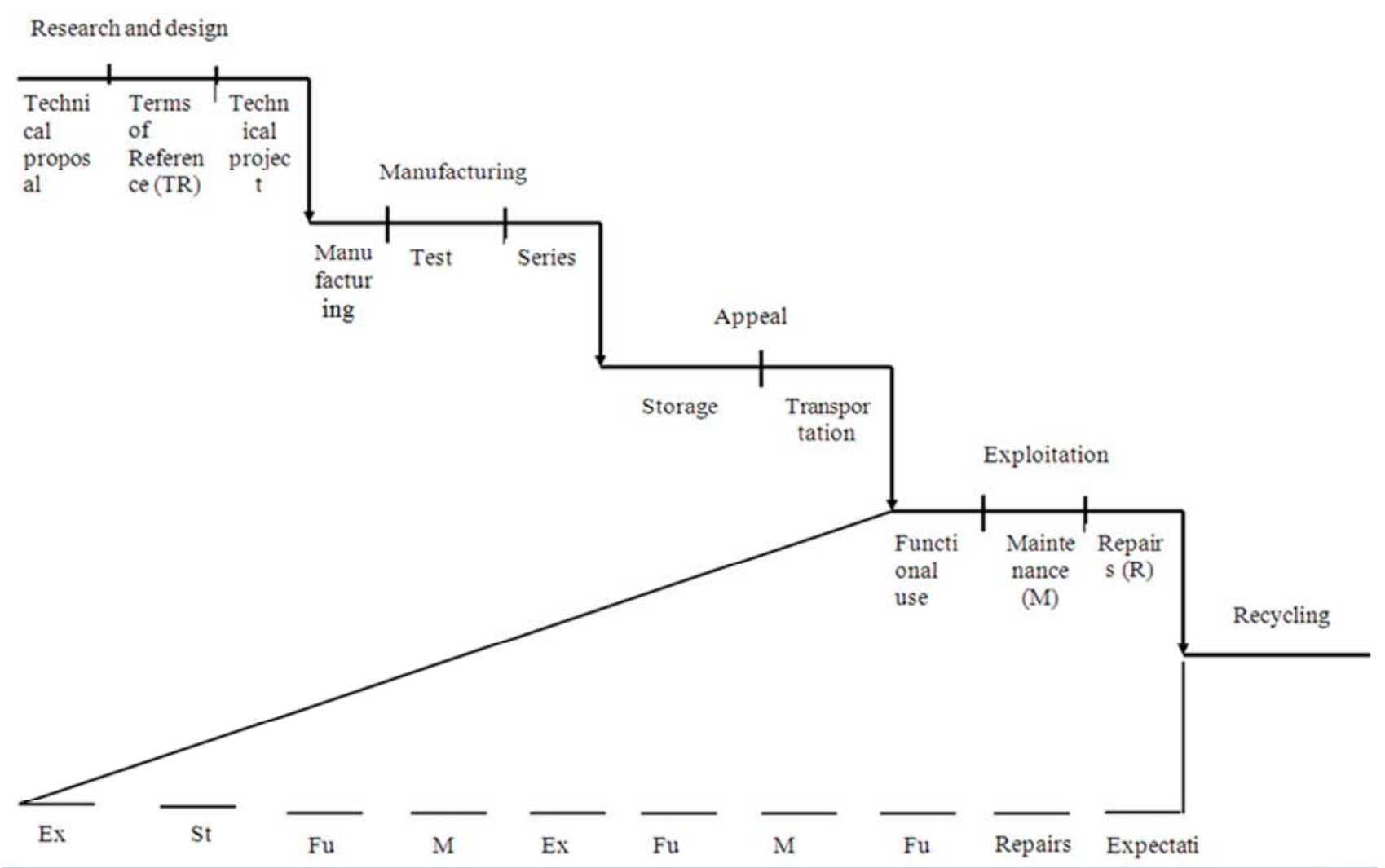

Figure 1. The life cycle of hardware and DTS in general.

The main criterion for reliability of the restored DTS is the availability factor $[3,8]$

$$
K_{\Gamma}=\frac{T_{H}}{T_{H}+T_{B}},
$$

which characterizes the probability that the system will be in a working state at an arbitrarily chosen time; where $T_{H}$ - mean time between failures;

This is the average value of the duration of the continuous operation of the system between two failures.

$$
T_{H}=\frac{1}{N} \sum_{i=1}^{N} T_{H_{i}},
$$

where N- total number of failures;

$T_{H_{i}}$ - time between $(i-1)$ and $i^{M}$ refusal. manufacturing, handling, operation and disposal of the system from the beginning of the study of the possibilities of its creation to the end of its intended use $[3,8]$.

In Figure 1 shows the typical distribution of stages and stages of the life cycle, DTS. We will consider the problems arising at the stage of the life cycle associated with the operation of DTS. So, the operation of DTS is the stage of the life cycle on which (functional use) is realized, maintenance (maintenance) is being maintained and its quality is being restored (technical maintenance and repair). 
Currently, the following main areas of work to improve the reliability of the operation of DTS are known:

1. First of all, reliability is enhanced by the use of highly reliable components. This direction is associated with significant expenditure of funds and provides only the solution of the problem of non-repudiation, but not maintainability. However, one-sided orientation in creating systems to achieve high reliability (through the use of a more sophisticated element base and nodes) to the detriment of maintainability, in many cases does not ultimately lead to an increase in availability in real operating conditions.

2. The second direction is connected with the improvement of operational and technical characteristics, DTS by improving the maintainability indicators by means of monitoring and technical diagnostics.

\section{Objectives and Classification of Systems of Technical Diagnostics of Microprocessor-Based DTS}

As noted earlier, the transition to a wide application of SIC, LIC and MPK in digital systems has created, together with indisputable advantages, a number of serious problems in their maintenance, primarily related to monitoring and diagnostic processes. It is known that the cost of troubleshooting at the production stage ranges from $30 \%$ to $50 \%$ of the total cost of device manufacturing, and at the operational stage of DTS, at least $80 \%$ of the recovery time of DTS is due to the search for a faulty replacement element [9-11].

Diagnostic systems DTS are designed to solve one or more problems: checking for serviceability; performance checks; verification of correct functioning; troubleshooting. In this case, the components of the diagnostic system are: the object of technical diagnosis, which is understood as an object or its components, the technical state of which is to be determined, the means of technical diagnosis, the set of measuring devices, the means of switching and coupling with the object.

Technical diagnostics (TD) is carried out in the system of technical diagnosis (STD), which is a collection of means and the object of diagnosis and, if necessary, performers, prepared for diagnosis and carrying it out according to the rules established by the documentation.

The components of the system are:

Object of technical diagnosis (OTD), which is understood as a system or its components, the technical condition of which is to be determined, and the means of technical diagnosis - a set of measuring devices, switching and coupling means with OTD.

Technical diagnosis system works in accordance with the algorithm TD, which represents a set of prescriptions for diagnosing.

The conditions for carrying out the TD, including the composition of the diagnostic parameters (DP), their maximum allowable minimum and maximum pre-stress values, the frequency of diagnosing the product and the operational parameters of the tools used, determine the mode of technical diagnosis and control.

Diagnostic parameter (attribute) is a parameter used in the established order for determining the technical state of an object.

Technical diagnosis systems (TDS) can be different in their purpose, structure, location, composition, design, circuitry solutions. They can be classified according to a number of characteristics that determine their purpose, tasks, structure, composition of technical means:

by the degree of OTD coverage; by the nature of the interaction between the OTD and the technical diagnostic and control system (STDK); on the means used for technical diagnosis and control; on the degree of automation OTD. [Figure 2] [3, 8]

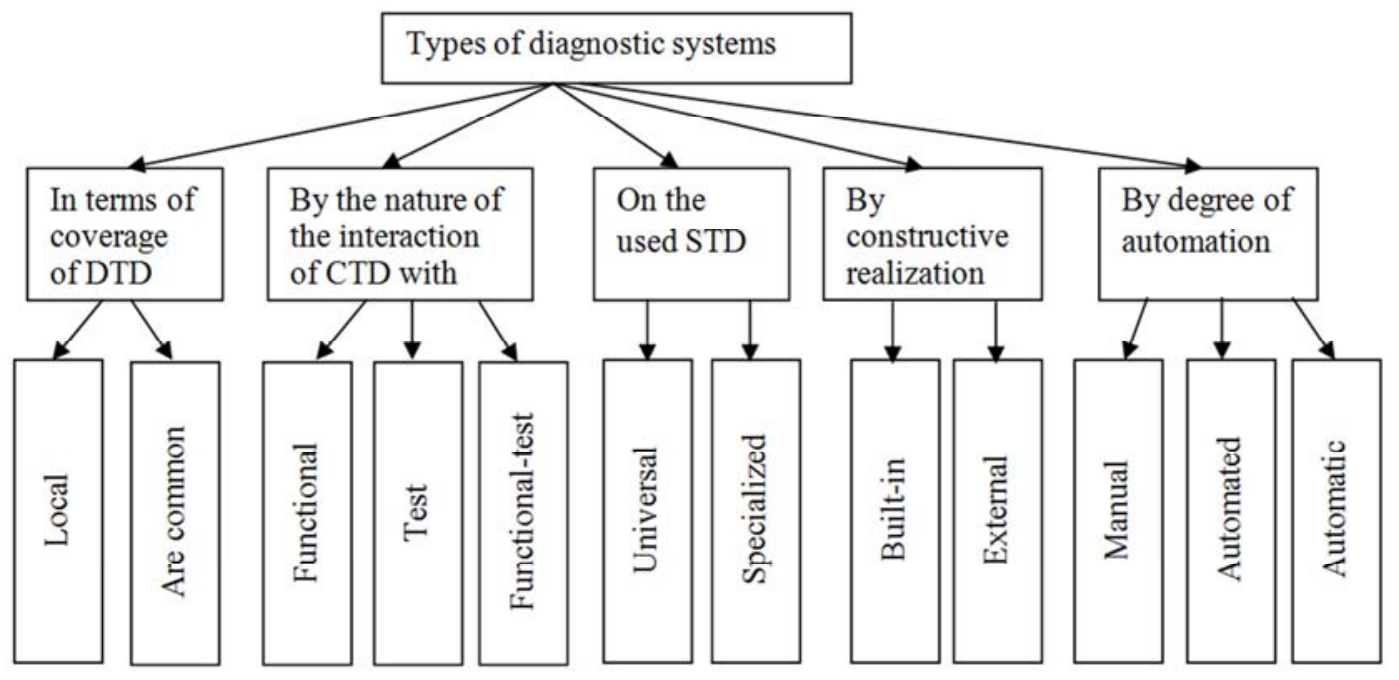

Figure 2. Types of diagnostic systems.

In order to maintain DTS in a technically sound condition, a monitoring and diagnostic subsystem is created, which is a 
set of software and hardware designed to diagnose their technical condition and maintain (or restore) the required quality of work. The DTS monitoring and diagnostic tools allow you to speed up complex processes for detecting and eliminating failures, and reducing equipment downtime.

\section{Analysis of Modern Microprocessor Devices (MPD) DTS as an Object of Monitoring and Diagnostics}

Increasing the technical and operational characteristics of complex DTS based on SIC, LIC and MPK is inextricably linked with the development of diagnostic methods and tools with the need for comprehensive accounting and analysis of digital cards and their components as an object of control and diagnostics.

Features of monitoring and diagnostics of digital cards with SIC and LIC are characterized by the following [3, 1214]:

(1) a wide range of SIC and LIC characteristics;

(2) the number of control tests, which can reach several thousand;

(3) digital cards with SIC and LIC have a trunking principle of organization, which requires the exchange of data on 4, 8, 16-bit buses in one clock cycle period, as well as simultaneous multichannel monitoring;

(4) trunks in most SICs and LICs have a bi-directional mode of operation, so the monitoring equipment must be able to switch from transmit to receive during one period of the clock;

(5) digital cards with SIC and LIC can have several bidirectional I / O channels in the interface circuits;

(6) since the time characteristics play an important role, the monitoring operations should be performed at a frequency close to the operating frequency to $10-50$ $\mathrm{MHz}$.

(7) Microprocessor devices have a number of features that prevent the use of traditional equipment:[9-14]

(8) the description of circuits is difficult, since their functions are realized in MPS by microprograms stored in ROM. The work of these schemes is hidden in the algorithm of the program;

(9) similar difficulties arise in connection with the dynamism of the operation of MPS, in which pulse signals usually act for several microseconds, and then disappear. Therefore, you need to know, not only where to look, but also when to look;

(10) the parallel structure of the buses to which several devices are connected at once according to the OR circuit, makes it difficult to detect a source of faults.

Thus, it is possible to point out the general features of digital cards based on SIC, LIC and MPK, which determine the complexity of their control:

(1) increased complexity of the control obje;

(2) limited admission to controlled nodes;

(3) bus organization;
(4) the need for real-time monitoring;

(5) microprogram control MP;

(6) incompleteness of control of SIC and LIC components;

(7) influence on the stability of the operation of the MPS of the input conductances SIC, LIC and structural elements;

(8) high cost of detection and elimination of defects, etc.

In order to develop methods and means of diagnostics, microprocessor boards and the creation of a diagnostic data base must be developed. The technique of the analysis of the nomenclature and technical data of the specified types of digital cards as a diagnostic object.

For this, it is necessary to analyze the nomenclature and technical data of digital cards and their components, which includes: [3, 8]

1. The distribution of the number of various digital cards in the digital system;

2. Number of typical names of digital cards and their sizes: types, series and number of SIC, LIC and MPK;

3. Types and number of connectors, the number of pins of connectors in various types of digital cards;

4. Operating frequencies of the functioning of the nodes in the digital cards in question;

5. Voltage grades of power supplies for various digital cards with IC, SIC, LIC and MPK.

Thus, the created diagnostic database is designed to store:

(1) information on the types of IMS, SIC, LIC and MPK and their reference signatures required for their replacement and for the organization of input control;

(2) information about the digital cards being checked and their reference signatures directly on the connectors' pins;

(3) information about the topological model of the circuit of digital cards;

(4) algorithms for searching and localizing the fault location in digital cards;

(5) information on external docking parameters required for setting up and checking the operability of the restored digital cards and bringing these parameters to the standards specified in the technical conditions.

\section{Methods for Calculating and Determining Reference Signatures for a Single and Multi-channel Signature Analyzer of Microprocessor Devices}

One of the types of diagnosing MPD at the stage of their development and manufacturing is the classical testing, the application of which allows to determine the correctness of their functioning and to carry out the procedure of troubleshooting. Testing of microprocessor circuits is based on the formation of test sequences, and during the testing procedure, based on the results of comparing real output reactions with the reference ones, decisions are made about the state of the circuit under test. If the received output reactions of the circuit are consistent with the reference 
scheme, it is considered to be functional, otherwise the circuit contains a malfunction and is in a faulty state. The classical approach to testing MPD requires considerable time-consuming both for the formation of test sequences and for the testing procedure. In this case, for carrying out the testing procedure, both the test sequences themselves and the standard output reactions of the schemes are stored for their effect.

Thus, the classic strategy of testing MPD, based on the formation of test sequences, requires significant time and large amounts of test information and standard output reactions, which implies the presence of complex equipment. The most effective solution, allowing to significantly reduce the amount of stored information about the reference output reactions, is a single and multi-channel signature diagnostic method in digital circuits. [4-7, 17]

An important parameter of the signature analysis is a set (dictionary) of reference signatures, which is predetermined for a functioning MPD. The need to create methods for calculating signatures is associated with the need to automate the production of dictionaries (tables) of reference signatures, since their creation by means of signature measurement is rather time-consuming. Therefore, the main document of the method of signature analysis is the dictionary of reference signatures, which defines an algorithm for troubleshooting. At the present time, various theoretical methods are known that allow one to calculate and determine reference signatures. $[15,16]$

\section{Method for Calculating Reference Signatures Based on the Nested Polynomial Method [16]}

Let the input binary sequence enter the input of the pseudo-random sequence generator (PSG) (Figure 3). PSG, similar to that shown in Figure 3, the input binary sequence is converted, corresponding to dividing the input polynomial by the inverse of the characteristic polynomial describing the given PSG.

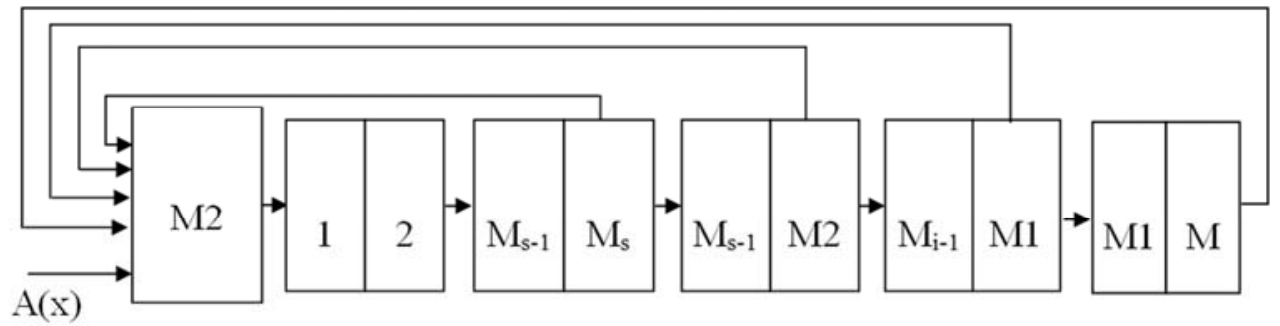

Figure 3. PSG scheme based on a shift register of lengths $n$ of digits.

Due to the fact that the numbering of registers usually starts from zero, the signature can be written in the form:

$$
G_{n}^{1}(X)=g_{m 1}(X) X^{m 1}+g_{m 2}(X) X^{m 2}+\ldots+g_{m s}(X) X^{m s}+r_{m s}(X) \text {. }
$$

To obtain a formula for calculating the signature in a particular PSG in expression (4) instead $X^{i}$, где $i=m_{1}, m_{2}, \ldots m_{s}$, it is necessary to substitute the digits of the PSG shift register, from which feedbacks are taken. For example, according to (4), for a PSG based on a 16-bit register, the formula for calculating the signature has the form:

$$
G_{16}^{H P}(X)=g_{12}(X) X^{12}+g_{9}(X) X^{9}+g_{7}(X) X^{7}+r_{7}(X) \text {. }
$$

Consider the PSG scheme for $n$ - bit shift register, with feedbacks taken from $n, m, l, k$ bits, which are put in the adder modulo 2. Then

$$
F(X)=g(X) \cdot P_{n}^{\prime}(X)+r(X),
$$

where $F(X)$ - input polynomial;

$$
\begin{aligned}
& g(X) \text { - quotient; } \\
& P_{n}^{\prime}(X)-\text { inverse feedback polynomial for a length } \\
& \text { gister } \\
& \mathrm{n} \text { - ranks; } \\
& r(X) \text { - remnant. }
\end{aligned}
$$$$
\text { register }
$$

Consider now the shift register, as a set of nested shift registers in one another $n, m, l, k$ ranks. It is obvious that each register performs its own division (transformation) of the input polynomial. If the degree of the input polynomial is greater than the degree of the shift register, then the effect of nested registers is taken into account in the feedback polynomial of the shift register of a larger length. If the degree of the input polynomial does not exceed the degree of the register, then the input polynomial divides into an inverse of the feedback polynomial of the shift register of a shorter length. Since the degree $r(X)$ less degree $P(X)$, then to obtain the real remainder it is necessary to divide $r(X)$ on inverse polynomials of feedbacks of registers of smaller length.

We denote the inverse inverse polynomial in terms of $P_{n}^{\prime}(X), P_{m}^{\prime}(X), P_{l}^{\prime}(X), P_{k}^{\prime}(X)$. We divide the input polynomial $F(X)$ on a polynomial $P_{n}^{\prime}(X)$ :

$$
\frac{F(X)}{P_{n}^{\prime}(X)}=g_{n}(X)+\frac{r_{n}(X)}{P_{n}^{\prime}(X)} \text {. }
$$


Then we look at the remainder $r_{i}(X),(i=n, m, l$,$) as input$ polynomials for shift registers of shorter lengths, dividing them by a polynomial $P_{i}^{\prime}(X),(i=n, m, l$,$) .$

$$
\begin{aligned}
& \frac{r_{n}(X)}{P_{m}^{\prime}(X)}=g_{m}(X)+\frac{r_{m}(X)}{P_{m}^{\prime}(X)}, \\
& \frac{r_{m}(X)}{P_{l}^{\prime}(X)}=g_{l}(X)+\frac{r_{l}(X)}{P_{l}^{\prime}(X)}, \\
& \frac{r_{l}(X)}{P_{k}^{\prime}(X)}=g_{k}(X)+\frac{r_{k}(X)}{P_{k}^{\prime}(X)} .
\end{aligned}
$$

Analysis of formulas $(4.5 \div 4.8)$ shows that $g_{n}(X)$ is an output polynomial of the degree register $n, g_{m}(X)$ - output polynomial of the degree register $m$, which fills the discharges from $m+1$ до $n$ shift register, $g_{1}$ - output polynomial of the degree register $l$, which fills the discharges from $l+1$ before $m$ and ect;

$r_{k}(X)$ - the remainder in the shift register, which has only one feedback and fills the bits from $l$ before $k$. Thus, the signature can be written in the following form $[3,4]$ :

$$
r_{c}(X)=g_{m}(X) X^{m+1}+g_{l}(X) X^{l+1}+g_{k}(X) X^{k+1}+r_{k}(X) X .
$$

By this formula, it is possible to calculate the signatures for the shift register with the numbering from $l$ before $n$. If the numbering of the digits starts from zero, then formula (4.9) takes the form:

$$
r_{c}(X)=g_{m}(X) X^{m}+g_{l}(X) X^{l}+g_{k}(X) X^{k}+r_{k}(X) .
$$

So, for PSG, developed by Hewlett Packard (HP) the formula has the form [14]:

$$
r_{c}^{H P}(X)=g_{12}(X) X^{12}+g_{9}(X) X^{9}+g_{7}(X) X^{7}+r_{7}(X) .
$$

Let's consider an example of calculation of a signature by the specified method for an input sequence 11111100000111111111, which we represent in a polynomial form:

$$
F(x)=x^{19}+x^{18}+x^{17}+x^{16}+x^{15}+x^{14}+x^{8}+x^{7}+x^{6}+x^{5}+x^{4}+x^{3}+x^{2}+x+1
$$

Dividing the input sequence $F(X)$ on the inverse polynomial $P^{\prime}(X)=x^{16}+x^{9}+x^{7}+x^{4}+1$, we obtain the: $g(X)=x^{3}+x^{2}+x+1 \quad$ and the remainder $r_{16}(X)=x^{15}+x^{14}+x^{12}+x^{11}+x^{7}$. Then we divide $r_{16}(X)$ on the inverse of the feedback polynomial of the 12-bit register $P(X)=x^{12}+x^{5}+x^{3}+1$. As a result, we obtain the $g_{12}(X)=x^{3}+x^{2}+1 \quad$ and the remainder $r_{12}(X)=x^{11}+x^{8}+x^{6}+x^{2}+1$. Dividing $r_{12}(X)$ on the inverse of the feedback polynomial of the 9-bit register $P_{9}^{\prime}(x)=x^{9}+x^{2}+1$. As a result, we obtain the $g_{9}(X)=x^{2}$ and the remainder $r_{9}(X)=x^{8}+x^{6}+x^{4}+1$.

Dividing $r_{9}(X)$ on the inverse of the feedback polynomial of the 7-bit register $P_{7}^{\prime}(x)=x^{7}+1$. As a result, we obtain the $g_{7}(X)=x$ and the remainder $r_{7}(X)=x^{6}+x^{4}+x+1$. Substituting these values into the expression for $r_{c}(X)$, we get

$$
\begin{aligned}
& r_{c}(X)=\left(x^{3}+x^{2}+1\right) x^{12}+x^{2} \cdot x^{9}+x \cdot x^{7}+x^{6}+x^{4}+x+1= \\
& x^{15}+x^{14}+x^{12}+x^{11}+x^{8}+x^{6}+x^{4}+x+1 .
\end{aligned}
$$

We transform, the resulting polynomial into a binary form $r_{c}(H P)=1101100101010011$, which corresponds to the signature H953. From the example given, it can be seen that this method of calculation requires the memorization of generators of polynomials in degree $n, m, l, k$, as well as additional calculations to obtain a real signature.

\section{The Method of Determining Reference Signatures Based on the Analysis of the Operation of a Single-Channel Signature Analyzer $[3,4,17]$}

As mentioned earlier, the principle of CA action is based on the compression of long sequences into four-digit, hexadecimal signatures. Physically, this method is implemented on a linear shift register with feedbacks, whose signals are summed modulo two with the input sequence. The polynomial is an irreducible polynomial $P(x)=x^{16}+x^{12}+x^{9}+x^{7}+1$.

Signatures are reproduced, as a rule, in an alphabet consisting of ten digits and six letters $0,1,2,3,4,5,6,7,8,9, \mathrm{~A}$, $\mathrm{C}, \mathrm{F}, \mathrm{H}, \mathrm{P}, \mathrm{U}$. Each binary sequence has its own signature.

0000 - "0" 0100 - "4" 1000 - "8" 1100 - "F"

0001 - "1" 0101 - “5” 1001 - “9” 1101 - "H”

0010 - "2" 0110 - “6” 1010 - "A" 1110 - "P"

0011 - “3” 0111 - "7" 1011 - "C" 1111 - "U”

In Figure 4 is a diagram explaining the principle of compressing an input sequence of length 20 bits by a 
signature analyzer.

The signature is formed using a shift register with a logical feedback $P(x)=x^{16}+x^{12}+x^{9}+x^{7}+1$, at the input of which there is an adder modulo two. Suppose that during the connection of the probe CA to a control point, a 20-bit sequence of ones and zeros appeared in it that has the form: 11111100000111111111 .
This input sequence is summed modulo 2 with the contents of the shift register cells 7, 9, 12 and 16. After 20 cycles of the circuit operation, the register will contain a 16-bit combination 1101100101010011, which, as a result of division into four four-digit combinations, corresponds to the alphanumeric signature $\mathrm{H} 953$.

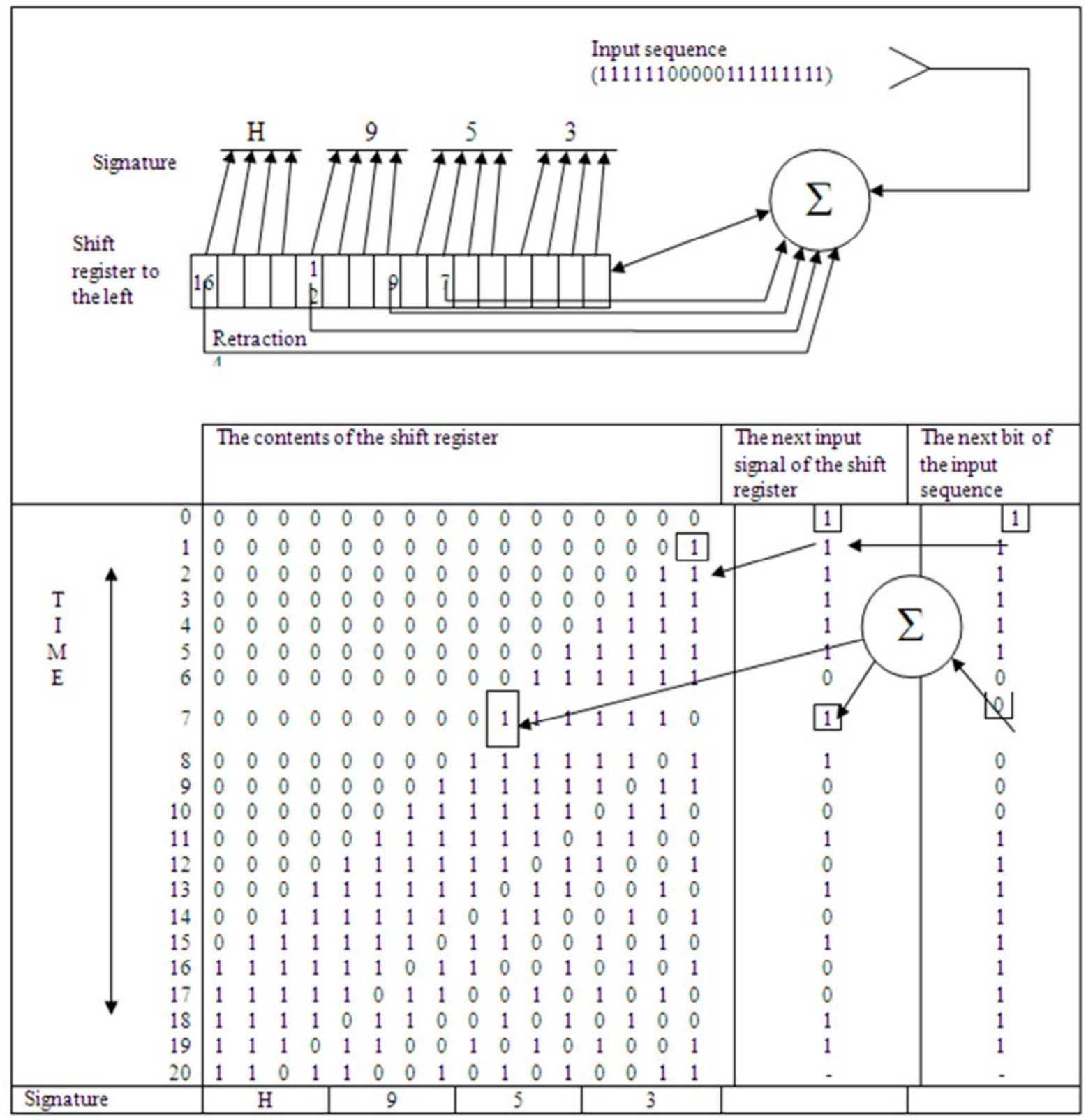

Figure 4. A diagram explaining the principle of compressing an input sequence of length 20 bits by a signature analyzer.

\section{A Simplified Method for Calculating Reference Signatures $[3,18]$}

As noted earlier, the principle of CA implementation as a whole is based on mathematical relationships similar to those used in the formation of cyclic codes. However, in view of the fact that the practical diagram of the divisor itself, performed on the multi-input adders modulo 2, differs from the used divisor of the cyclic code, the contents of the shift register $\mathrm{CA}$ and the result of division $F(X)$ on $P(X)$ do not match. It is known that the remainder obtained in the shift register has $R(x)=x^{15}+x^{14}+x^{12}+x^{11}+x^{8}+x^{6}+x^{4}+x+1$, and the remainder upon division $F(X)$ on $P^{\prime}(X)$ has the form: $\mathrm{r}_{16}(\mathrm{X})=x^{15}+x^{14}+x^{12}+x^{11}+x^{7}$.

In this connection, it is necessary to analyze not the 
remainder of fission, but the quotient from fission. In accordance with this technique, the signature is calculated by multiplying the input polynomial $F(X)$ on a monomial $X^{r}$ and dividing this product by the inverse generator polynomial $P^{\prime}(X)$ :

$$
\frac{F(X) X^{r}}{P^{\prime}(X)}=Q(X)+\frac{R(X)}{P^{\prime}(X)} \text {. }
$$

In this case, the quotient of the division has the same degree as $F(X)$, and the signature is the last 2 digits. $r_{c}(X)=[\mathcal{Q}(X)] \bmod 2^{r}$.

Let us consider an example of a theoretical calculation of the signature by this method for a similarly-considered input sequence. Multiplying the input polynomial $F(X)$ on a monomial $X^{16} \quad, \quad$ we get: $F(X) X^{16}=x^{35}+x^{34}+x^{33}+x^{32}+x^{31}+x^{30}+x^{24}+x^{23}+x^{22}$. $+x^{21}+x^{20}+x^{19}+x^{18}+x^{17}+x^{16}$

We divide this polynomial by the inverse polynomial $P^{\prime}(X)=x^{16}+x^{9}+x^{7}+x^{4}+1$.

As a result, we obtain the

$\mathrm{Q}(\mathrm{X})=\mathrm{x}^{19}+\mathrm{x}^{18}+\mathrm{x}^{17}+\mathrm{x}^{16}+\mathrm{x}^{15}+\mathrm{x}^{14}+\mathrm{x}^{12}+\mathrm{x}^{11}+\mathrm{x}^{8}+\mathrm{x}^{6}+$ $\mathrm{x}^{4}+\mathrm{x}+1$ and the remainder $R(X)=x^{14}+x^{9}+x^{8}+x^{7}+x^{6}+x^{5}+x+1$.

Transforming $Q(X)$ in the binary form we get $\mathrm{Q}(\mathrm{X})=11111101100101010011$

The last 16 digits are a signature, i.e. $\xi \underset{c u}{H P}$ $(\mathrm{X})=1101100101010011$ (H953).

\section{The Method for Determining Signatures Based on the Simulation of a Single and Multi-channel Signature Analyzer [19, 20]}

The program for simulating a single-channel signature method for diagnosing digital devices SIGNATURA is designed to visually display the process of forming a digital signature when diagnosing a device. The program simulates the process of passing a digital sequence through a logic circuit consisting of a shift register and an adder modulo 2 . After the simulation process is over, the program outputs the result - a digital signature in the form of a code for displaying on a seven-segment indicator. The program works in the operating systems Microsoft Windows NT / 2000 / XP / 2003/7/10.

The program does not require installation and is launched by running the signatura.exe application. When you start, the program window appears.

The program window contains the image of the simulated circuit in the upper part, the control elements at the bottom.

The sequence of work with the program is as follows:

(1) set the shift register;

(2) set bits of a polynomial;

(3) input input sequence;

(4) If necessary, set the interval for acceleration / deceleration of the process;

(5) click the "Start" button.

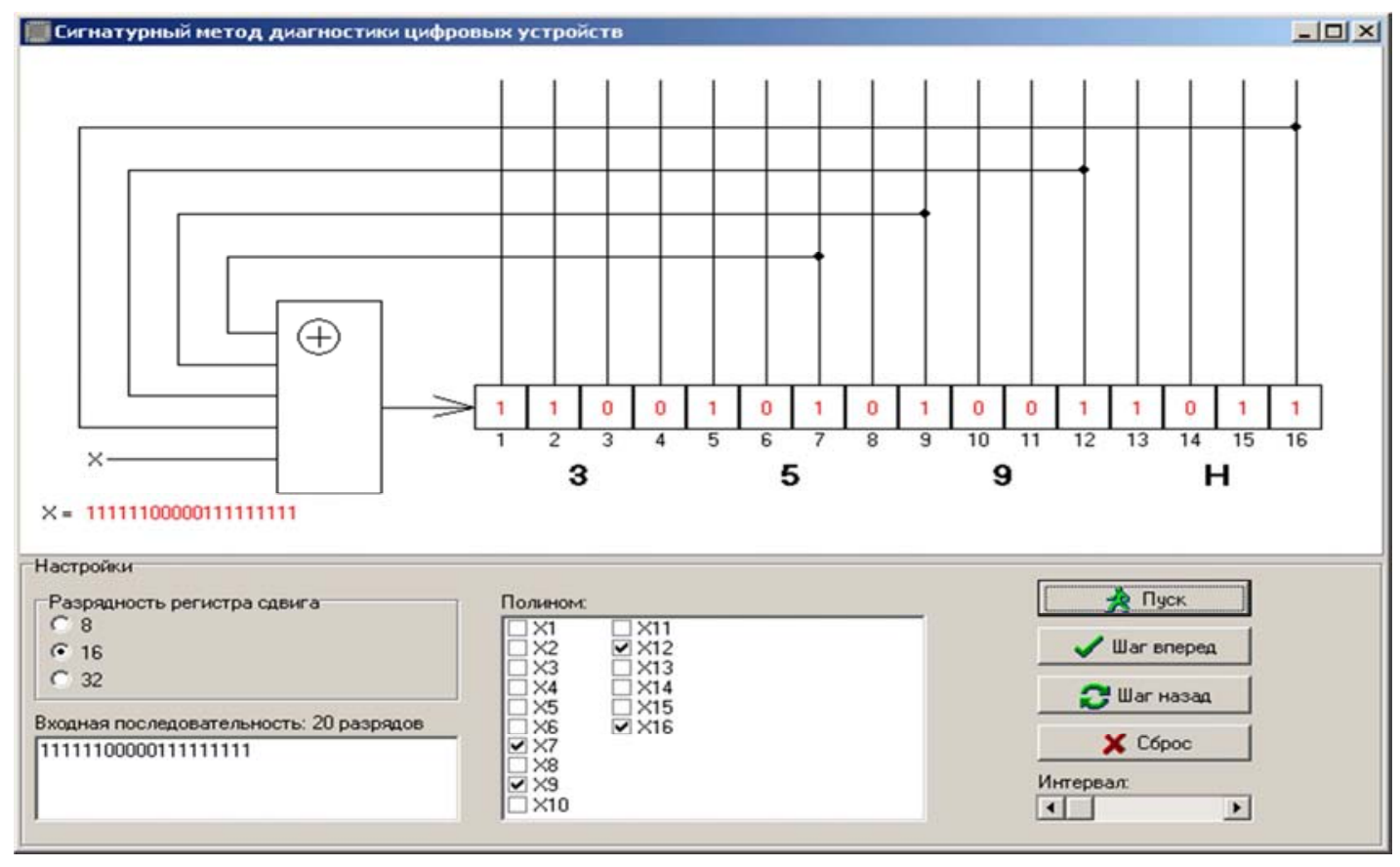

Figure 5. Digital signature after the end of the process.

When the "Start" button is pressed, the input sequence starts to be fed to the input of the adder modulo 2. The bits of the input sequence pass through the logic circuit and are written to the shift register. 
After passing through all the bits of the input sequence through the logic circuit, a digital signature appears on the screen, representing the code for the seven-segment indicator (Figure 5).

Each signature symbol is displayed under the corresponding four bits of the shift register. A six-digit register corresponds to a four-character signature, an eight-digit two-character signature, a thirty-two-digit eight-digit signature.

An analysis of the n-output digital circuit by a single- channel signature analyzer (CA) leads to an increase in $n$ times of the time required for circuit analysis or equipment required to implement $\mathrm{n}$ signature analyzers. Multichannel signature analyzers allow to significantly speed up the procedure for monitoring multi-output microprocessor circuits, which practically increases by $n$ times, where $n$ is the number of inputs of the analyzer used.

Description of the virtual mock-up of a multichannel signature analyzer.

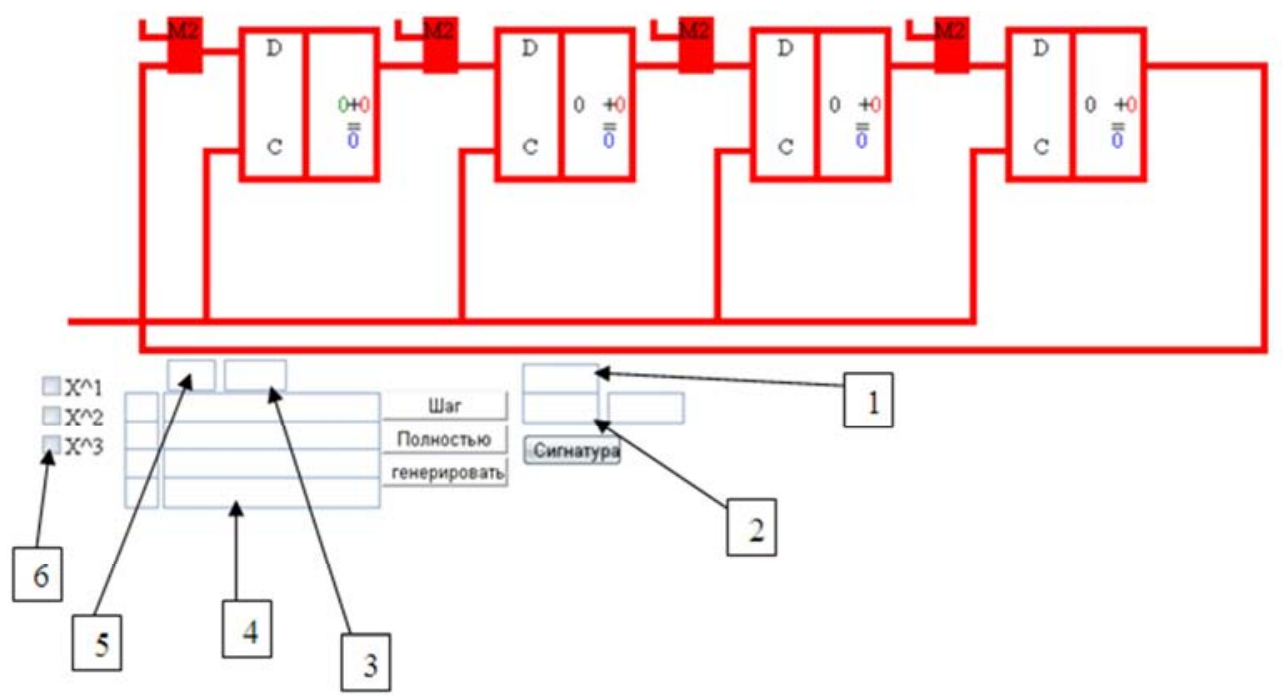

Figure 6. Multi-channel signature analyzer.

(1) the state of the triggers in the last step of the operation.

(2) convolution (signature).

(3) incoming vector.

(4) field for displaying sequences.

(5) fields for entering the length of the sequence.

(6) field for register feedback.

Symbols of red color - the next incoming bit vector.

Symbols of black color-the state of the previous triggers in the previous bar.

Green symbols - the state of the last trigger in the previous bar (appears when there is feedback).

Symbols of blue color - trigger states in the current bar.

In order to get the convolution, you must first enter the length of the stream (field \# 5) then click on the "generate" button and then on the "calculate" button during the last clock of the analyzer operation, in field \# 1 (the state of the triggers in the last step of the operation) there will be a binary sequence which is not yet a minimized signature, then when the "signature" button is pressed in box \# 2, the final convolution appears. Also it is possible to establish feedbacks in field No. 6.

In addition, you can enter the desired sequences yourself, for this you need to specify the length of the sequence in the field for specifying the length (field \# 5), and in the sequence mapping field (field \# 4), we introduce sequences.

This program works on all operating systems supporting browsers such as Opera, Mazilla firefox, Explorer.

The figure shows the interface of the program for modeling the operation of a multichannel signature analyzer for 4, 8, 16 bits (channels).

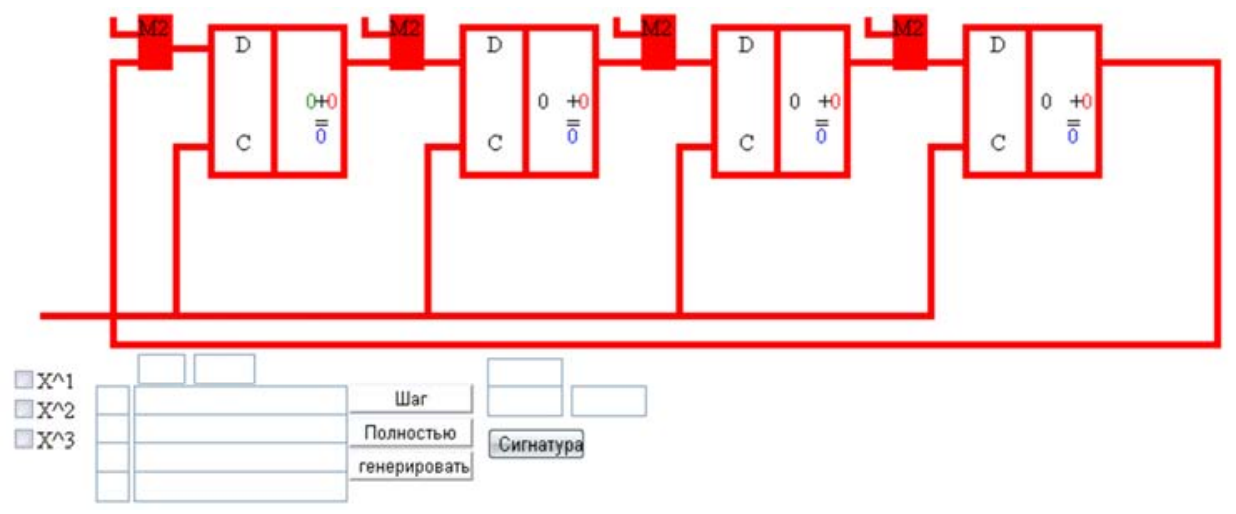

Figur 7. 4-channels of the signature analyzer. 


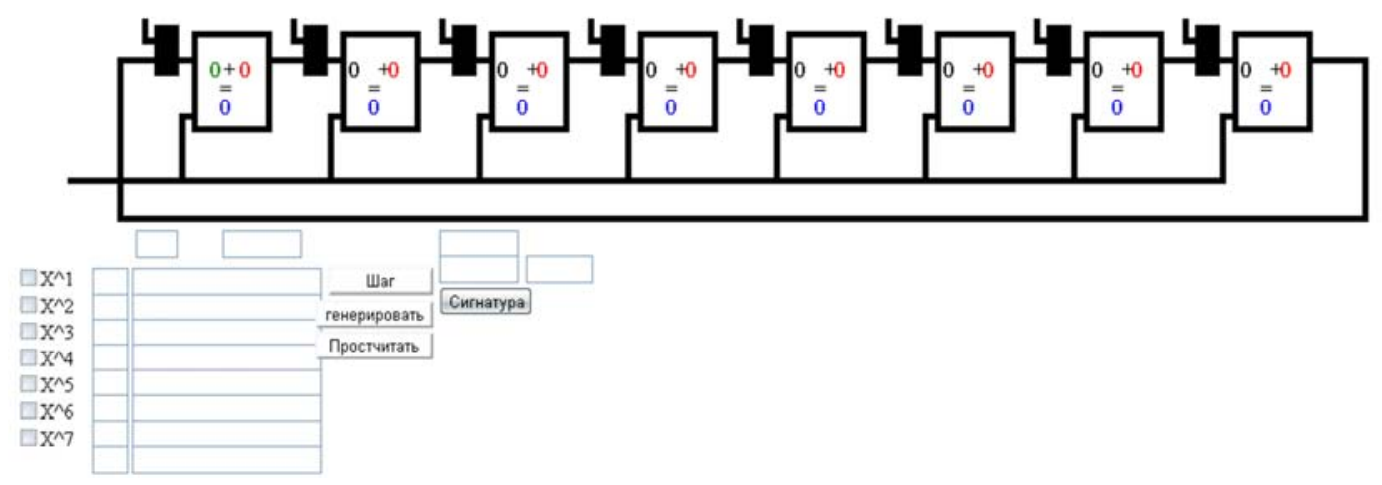

Figure 8. 8-channels of the signature analyzer.
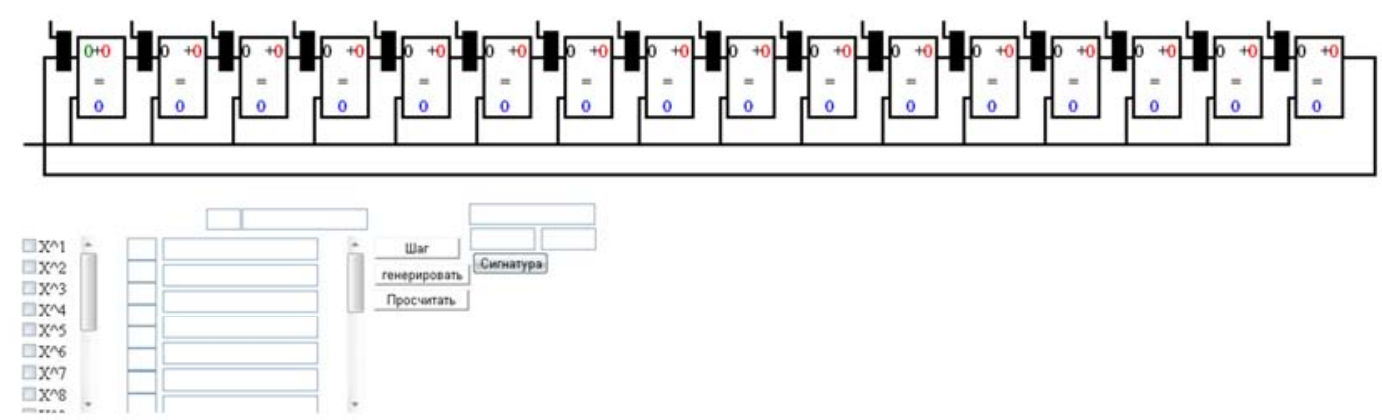

Figure 9. 16-channels of the signature analyzer.

These figures represent the time of the model's operation at a certain time.

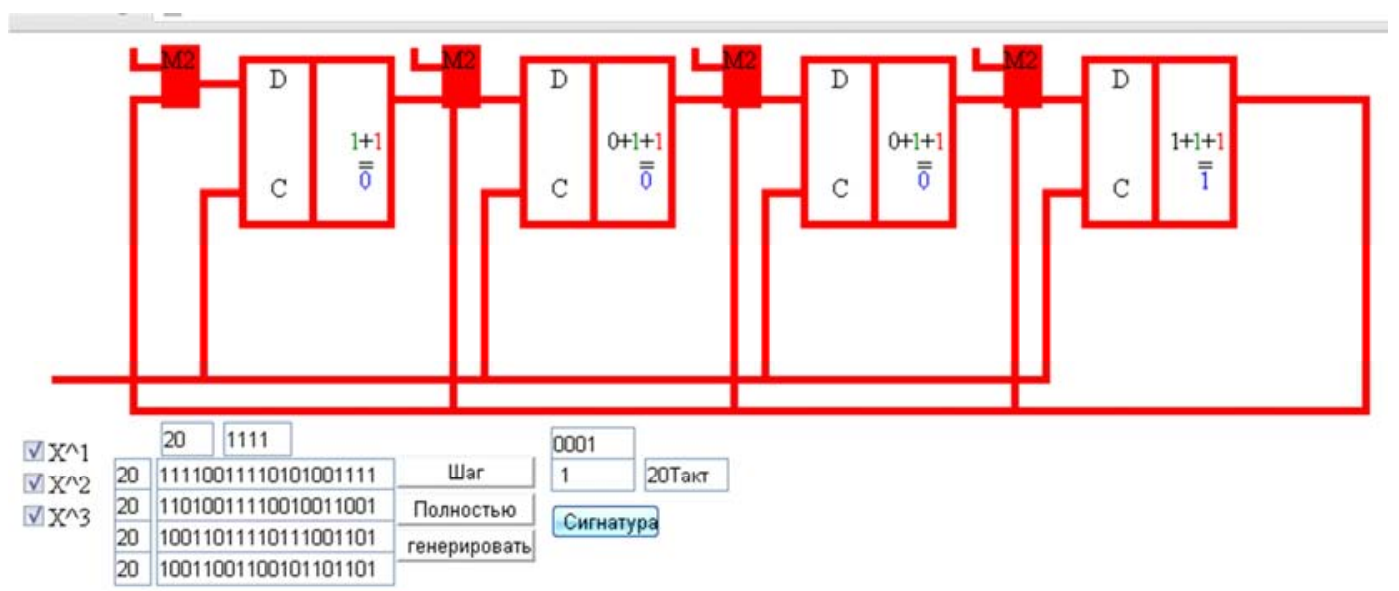

Figure 10. 4-channels of the signature analyzer.

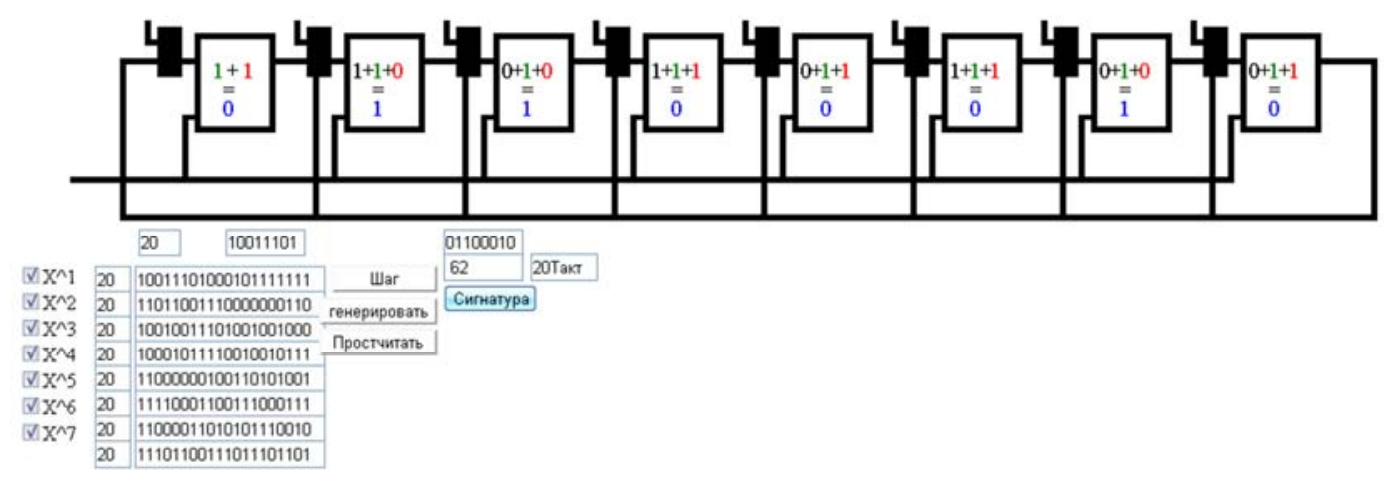

Figure 11. 8-channels of the signature analyzer. 

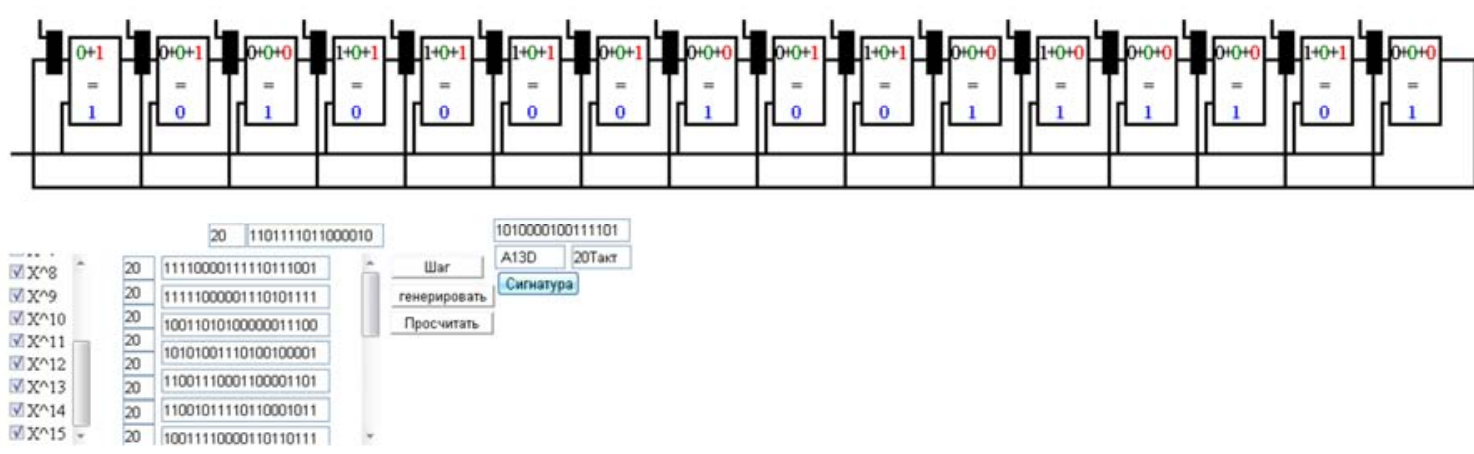

Figure 12. 16-channels of the signature analyzer.

\section{Conclusion}

Based on the studies carried out, the following conclusions can be drawn:

A scientifically substantiated solution of complex monitoring and diagnostic tasks DTS, using SIC, LIC and MPK kits in its composition, can be provided on the basis of a systematic approach to the development of control and diagnostic support for various stages of the life cycle.

A technique for analyzing DTS microprocessor devices as a monitoring and diagnostic object for selecting characteristics of the signature analysis method.

The general methodology of the use of signature analysis presupposes the work and the definition of reference signatures for a known microprocessor device, analysis, therefore, the existing mathematical methods of calculation and determination of reference signatures.

For the purpose of automating the definition of reference signatures, algorithms and programs have been developed for simulating single and multichannel signature analysis that allows to change both the length of the shift register and the types of irreducible ones that allow to change both the width of the shift register and the types of irreducible polynomials and the lengths of the analyzed binary sequences.

The developed software for simulating single-channel signature analysis runs on Microsoft Windows NT / 2000 / XP / 2003/7/10 operating systems, and the program for simulating the work of multi-channel signature analysis is written in PHP and using the Devel Studio program.

\section{References}

[1] AR Bestugin, AF Bogdanova, GV Stogov. Monitoring and Diagnostics of Telecommunication Networks - $\mathrm{SPb}$ : Politechnica, 2003. 174 p.: silt.

[2] Aripov MN, Prisyazhnyuk SP, Sharifov RA Control and management in data networks with packet switching. Tashkent.: Fan, 1988. - 160p.

[3] Aripov M. N. Juraev R. Kh., Jabbarov S. Yu. Technical diagnostics of digital systems. Training Manual Tashkent 2006, p.82.

[4] Abramovici M, Breuer M. A, Friedman A. D. Digital Systems
Testing and Testable Design. IEEE Press, Piscataway, New Jersey, 1994.

[5] Zainalabedin Navabi, Digital System Test and Testable Design: Using HDL Models and Architectures, Springer, 2010.

[6] Moslem Amiri, Vaclav Prenosil. Digital Systems Testing. Verilog HDL for Design. a Embedded Systems Laboratory. Faculty of Informatics, Masaryk University. Brno, Czech Republic. 2014.

[7] N. K. Jha and S. Gupta. Testing of Digital Systems. Cambridge University Press 2003.

[8] Davydov P. S. Technical diagnostics of radio electronic devices and systems. -M.: Radio and communication, 1988. - $256 \mathrm{~s}$.

[9] Microprocessors: Programming and debugging systems / VA Myasnikov, MB Ignatiev, AA Kochkin, Yu. E. Sheinin / Under red.VA Myasnikova and MB Ignatieva. -M.: Energoatomizdat, 1985. $-272 p$.

[10] Khakhanov V. I. Technical diagnostics of digital microprocessor devices. Textbook. K.: IZMN, 1995. - 252p.

[11] Lvovich G. A., Samoshchenko A. V., Khavkin V. E. Diagnostics of microprocessor systems, - Reviews of electronic equipment. Ser.3. Microelectronics, issue 7. - Moscow: TsNII Electronics, 1987. - 84s.

[12] Williams GB Debugging of microprocessor systems: Trans. with English.- M.: Energoatomizdat, 1988.-253p.

[13] Ferguson J, Makari L, Williams P. Servicing of microprocessor systems: Trans. with the English.- M.: Mir, 1989. - 336s.

[14] Djeykoks Dj. Guide to troubleshooting electronic equipment. Translation from English. - Moscow: Mir, 1989. - 176s.

[15] Kazmina S. K. Compact testing // Automation and telemechanics, 1982, N3, p.173-187.

[16] Gulyaev V. A., Kudryashov V. I. Automation of adjustment and diagnostics of microUK.-M.: Energoatomizdat, 1992.256 s.

[17] Gordon G., Nadig H. Localization of malfunctions in microprocessor systems with hexadecimal key codes. // Electronics.1977, N5, p.93-97.

[18] Djabbarov Sh. Yu., Djuraev R. X., Baltaev J. B. CONFERENCE PROCEEDINGS MITA 2015. June 30-July 2, 2015, Tashkent, Uzbekistan. "Simulation of the multichannel signaling method for diagnosis in multimedia terminal devices". 139-142. 
24 Rustam Xusanovich Djuraev and Jo'shqin Boltabaevich Baltaev: Investigated Methods of Improving the Efficiency of Diagnosing Microprocessor Devices of Data Transmission Systems Based on Multi-Channel Signature Analysis

[19] Juraev R. Kh., Jabbarov S. Yu., Yangaliev F. Sh., Program for modeling the signature method of diagnostics of digital devices. // No. DGU 01244, 26.03.2007.
[20] Juraev R. Kh., Jabbarov S. Yu., Baltayev JB, Program of modeling of four-channel, eight-channel and sixteen-channel signature method of diagnostics of digital devices. // No. DGU 04122, No. DGU 04123, No. DGU 04124 08.11.2016. 